\title{
Student Evaluation of Nursing Simulation Lab Learning Experience
}

\author{
Kawther Hamash ${ }^{1 *}$, Jeong IL Cho ${ }^{2}$, Koichiro Otani ${ }^{3}$, Yihao Deng ${ }^{4}$ and Erika Klingler ${ }^{5}$ \\ ${ }^{1}$ School of Nursing, Indiana University School of Nursing Fort Wayne, USA \\ ${ }^{2}$ Department of Education, Purdue University Fort Wayne, USA \\ ${ }^{3}$ School of public health policy, Purdue University Fort Wayne, USA \\ ${ }^{4}$ Department of Mathematical Sciences, Purdue University Fort Wayne, USA \\ ${ }^{5}$ School of Nursing, Indiana University School of Nursing Fort Wayne, USA
}

*Corresponding author: Kawther Hamash, Assistant Professor, School of Nursing, Indiana University Fort Wayne, USA.

Received Date: June 29, 2020

Published Date: July 16, 2020

\begin{abstract}
Objective: Describe and examine the effect of influential factors in simulation labs on the students' total experience in learning the concepts taught in the didactic portion of nursing courses at a four-year university.

Background: Nursing education is developing leading to a great emphasis on new, effective strategies that focuses on using simulation labs to enhance students' clinical judgment and critical thinking. The end of semester student evaluation of teaching (SET) of simulation labs reflect factors that can affect the students' learning experience. SET of simulation labs helps faculty plan for future changes in course objectives based on the students' needs.

Method/design: Two hundred and eighty-five anonymous student evaluations of teaching simulation lab surveys were collected retrospectively for the academic years of 2014 through 2016.

Results: Multiple regression analysis showed that the most influential item was "Lab experiences clarify the lecture material." The least influential item was "I can complete the lab activities in the time allotted." Students were mostly concerned about how the simulation lab will prepare them to better understand the associated didactic course material and concepts. Students' evaluations were also affected by the availability and quality of simulation equipment used in the simulation lab.

Conclusion: Students' evaluation of simulation provided input about factors that can affect how and what students are learning during simulation time. Using students' input in the evaluation of the simulation lab will help nurse educators strengthen the nursing curriculum and make necessary changes to meet the course objectives, students' needs, and external learning outcomes.
\end{abstract}

Keywords: Simulation; Nursing; Influential factors; Student evaluation; Learning; Clinical

\section{Introduction}

Nursing education is changing which places a greater emphasis on enhancing effective teaching strategies used including didactic, clinical, and simulation labs [1]. Recently, healthcare has recognized the importance of using simulation labs to enhance students' clinical judgment. The current healthcare trends are directing toward encouraging the use of simulation labs in nursing education [2]. Nursing simulation labs provide nursing faculty the ability to expose students to real-life acute care scenarios that 
allow students to learn how to provide safe nursing care in such a setting once they become a Registered Nurse (RN). Simulation labs also help the students develop nursing skills and build clinical reasoning abilities that focus on patient outcomes [3]. Simulations facilitate the introduction of static nursing skills that are usually demonstrated by using case studies and computer-generated scenarios [4,5]. Additionally, simulation labs benefit students by allowing them to manage a variety of clinical situations in a safe learning environment before attending to actual patients [6].

Identifying the outcomes planned for simulation labs at the beginning of each academic year is critical for the successful implementation of needed changes that were identified in the student evaluations and feedback. Each simulation course's outcomes need to be integrated with the didactic course outcomes and objectives to ensure consistency between both courses. Also, simulation objectives should address/evaluate the integration and application of knowledge learned, critical thinking, clinical judgment, and prioritization in each nursing course and students involvement in the evaluation of the simulation labs is necessary as they measure learning outcomes and course objectives at the end of the simulation course [7].

Students provide feedback at the end of each semester to evaluate their learning experience in simulation labs. Student feedback is a rich source for evaluating their learning experience and addressing students' needs in future simulation sessions. A student's comfort, confidence, and satisfaction level at the end of each simulation session is important and should be used as an ongoing assessment to identify changing needs that emerge during the implementation of simulation labs and used to improve students' learning experience [3]. It is recommended to evaluate a course's learning outcomes from the student's perspective to enhance their learning experience [7]. Students' evaluation and feedback collected at the end of each semester can be used as a tool to plan for future changes that can address the students' needs and the nursing program's outcomes. Thus, the evaluation of simulation lab experience can be a good source of information for instructors to use to enhance the students' learning experience. Multiple factors can influence the students' evaluation of their learning experience. These factors were categorized into students' background/ characteristics, faculty characteristics, school characteristics, and use of learning strategies [8]. In this study, we focused on the school and lab characteristics, and the characteristics of the instructor.

\section{Role of supplies}

Simulation labs in the nursing field are equipped with different fidelity level mannequins and computerized systems to run roleplaying scenarios that mimic real-life patient situations. Simulation equipment is a resource that can be used to facilitate the learning experience for students with different learning styles. High quality, also called high fidelity, simulation resources allow students to have real-life experience in a safe learning environment. Mannequins are controlled by computer equipment to allow students to practice different essential skills $[9,10]$. High fidelity simulation mannequins are often expensive which means offering true-life scenarios may be an obstacle for schools with a low simulation budget. Lower fidelity mannequins do not offer the same quality of student learning experience compared to new, higher fidelity mannequins. Thus, the quality of supplies provided in simulation labs can influence students' learning experience.

\section{Role of the instructor and use of time}

A simulation is run by a facilitator who can be the course lead instructor or a graduate student assisting the lead instructor. The role of the facilitator is important in the students' simulation learning experience. The facilitator's role is managing and organizing the simulation sessions as well as provide effective feedback and debriefing toward the end of each simulation session. Facilitators play a key role in managing the students learning experience by providing a comfortable environment and effective time management techniques. The simulation facilitator is responsible for helping the students apply the knowledge gained from different clinical scenarios to make safe clinical judgments and build the basic and advanced skills required to care for patients in the clinical setting $[7,11,12]$. The instructor's preparedness and effective use of simulation lab time can change the students' perspectives about the effectiveness of simulation labs. Thus, instructors need to integrate the students learning outcomes in the didactic courses with the simulation lab activities to ensure its congruency with the simulation lab learning outcomes.

Simulation sessions are usually planned by the facilitator to meet the course objectives. Simulations run by the instructor offers the students the chance to receive ongoing feedback to correct their actions to promote the patient's outcomes $[4,13]$. At the end of a simulation, students engage in a debriefing where they can ask questions, discuss actions are taken and address actions that could have been done differently which allows the students to think more critically of their actions performed and clinical judgments made during the simulation and discuss the impact of said actions and what could be done differently [13]. On the other hand, simulations run by assisting students are associated with some limitations. Student planned simulations are planned on multiple dates with the facilitating student simulating a remote room and the students gather at the end of the simulation for debriefing. Students will not receive ongoing feedback or cues while the simulation session is running [13]. This difference highlights the students' needs as reported in their evaluation of simulation labs and emphasizes the need to use the students' evaluations in building plans to change the approach of running simulations in nursing labs $[7,14]$. The student summaries of labs and course evaluations serve different purposes that can be used for program growth and decisions related to the accreditation process [1]. The success of accredited nursing programs is measured using a comparative measure such 
as the NCLEX-RN examination. Students' evaluation of lab and course evaluation can assist in improving the annual success rates in this competency examination [1].

\section{Tools used for student evaluation of simulation lab learning experience}

The type of tools used in students' evaluations of nursing simulation labs was inconsistent. The literature revealed that most of the nursing studies focusing on simulation labs used different evaluation tools and measured a different outcome. For example, Lewis and Ciak [15] used the National League for Nursing (NLN) to measure satisfaction and self-confidence to evaluate students learning and confidence levels after implementing simulation labs. In another study, Portsman, et al. [3] used the Psychomotor Skills Performance subscale (PSP) and the Clinical Competency Appraisal Scale (CCAS) to evaluate the students' competency of the skills learned in simulation. Jeffreys, et al. [1] used multidimensional tools for student evaluation, and Casida and Shapkof [11] used a threedimensional tool in a retrospective study to evaluate students perception of simulation teaching. The tool reflects the cognitive, psychomotor, and affective domain of students' learning [11]. There is no standardized tool used for evaluating the simulation lab experience, thus the focus on the outcome measure, and the evaluation tool was inconsistent across the different studies.

Based on Speaking of Teaching [16] evaluation tools should have specific areas the students evaluate their instructor's ability to engage them in the simulation activities and how well the simulation activities were integrated into the didactic course objectives. Speaking of Teaching [16] has suggested four target areas that need to be addressed in students' evaluations to help improve faculty teaching strategies. The first target is to state and relate the course objectives of the simulation lab sessions with the objectives of the didactic course. The second target is the presentation of the course material at an appropriate pace that covers the concept of each session. The third target is assisting students in developing and enhancing their conceptual understanding and critical thinking skills and the final target is the incorporation of course assignments to focus on problem-solving and the application of existing knowledge and provides a summary from the time spent in the simulation lab [16]. Although students' evaluations of simulation labs are important in enhancing their learning experience, discrepancies in the measurement of outcomes and evaluation tools used in different studies will limit the benefit of the prior study results. Instructors are unable to find prior research studies that provide them with recommendations on how to use students' evaluation of simulation courses to make future changes. To help instructors improve their teaching of simulation labs and use of student's evaluations to enhance their student's learning experience, the current study is planned to identify factors that can influence student positive learning experience in a simulation lab at a four-year university.

\section{Material and Methods}

\section{Data collection and instrument}

The purpose of this study is to describe and examine the effect of influential factors in simulation labs on the student's total experience in learning the concepts taught in the didactic portion of nursing courses in the department of nursing in a Midwestern university. Institutional review board approval was obtained before collecting retrospective data. The simulation lab evaluation forms were administered online in the nursing department by Class Climate (CC) in the academic years of 2014 through 2016.

The CC survey includes items that focus on the simulation lab learning experience and the survey is conducted at the end of each semester. Responses to CC survey questions are recorded anonymously and student responses are kept at a university teaching and learning center to protect the confidentiality of the course faculty and the students. Students are encouraged to complete the online survey objectively. Survey items are measured on a Likert type scale ranging from [1] "strongly disagree" to 5 "strongly agree". To maintain the confidentiality of the instructor and student information, the evaluation dataset collected represents a de-identified set of student responses to the evaluations of nursing simulation labs. The data was saved in an Excel worksheet and converted to SPSS file for data analysis. The survey used by the department evaluated students' ratings of lab course evaluation items. The tool includes seven items that measure different aspects of students' lab experience. The seven questions assess the students' evaluation of their simulation laboratory experience. The eighth question measures the students' overall experience with the simulation lab. The evaluation questions were developed based on the course objectives from a valid set of items that were developed and recommended by the university.

Multiple linear regression analysis was used to identify the effect of seven aspects of simulation lab learning on the students' experiences in learning concepts. The dependent variable (DV) is "the lab experiences assist me in learning concepts" while the independent variables (IVs) are the attributes of the seven items collected in student evaluations.

\section{Findings}

Table 1: Descriptive mean scores of student's lab experience detailed by items.

\begin{tabular}{|c|c|c|c|}
\hline Variable Description & N & Mean & SD \\
\hline Lab procedures are clearly explained to me & 285 & 4.18 & 0.954 \\
\hline The lab in this course has adequate facilities & 285 & 4.39 & 0.721 \\
\hline
\end{tabular}




\begin{tabular}{|c|c|c|c|}
\hline The content of the lab is a worthwhile part of this course & 285 & 4.25 & 0.989 \\
\hline My instructor is able to answer my questions about what I should be doing in the lab & 285 & 4.3 & 0.868 \\
\hline I am able to complete the lab activities in the time allotted & 285 & 4.32 & 0.818 \\
\hline The lab sessions are well organized & 285 & 4.04 & 1.013 \\
\hline Lab experiences assist me in learning concepts* & 285 & 4.34 & 0.831 \\
\hline
\end{tabular}

$\left.{ }^{*}\right)$ Dependent variable item in the scale.

Table 2: Multiple regression analysis of factors influencing student's lab experience in learning course concepts.

\begin{tabular}{|c|c|c|c|c|}
\hline Item & Item Rank & B & SE & $P$ value \\
\hline (Constant) & - & 0.098 & 0.139 & 0.479 \\
\hline Lab experiences clarify the lecture material & $1 \mathrm{st}$ & 0.297 & 0.054 & 0 \\
\hline The lab in this course has adequate facilities & 2nd & 0.188 & 0.046 & 0 \\
\hline The content of the lab is a worthwhile part of this course & 3rd & 0.162 & 0.031 & 0 \\
\hline Lab procedures are clearly explained to me & 4 th & 0.158 & 0.049 & 0.001 \\
\hline My instructor is able to answer my questions about what I should be doing in the lab & 5 th & 0.134 & 0.054 & 0.013 \\
\hline I am able to complete the lab activities in the time allotted & 6th & 0.112 & 0.042 & 0.008 \\
\hline The lab sessions are well organized & 7 th & -0.06 & 0.043 & 0.166 \\
\hline $\mathrm{R} 2=0.816$ & & & & \\
\hline
\end{tabular}

Statistical analysis was performed using SPSS version 24. This study included data from 285 de-identified course evaluations of baccalaureate undergraduate nursing students. Of these, 248 were female students and 37 were male students. Around $63.5 \%$ of the sample was enrolled full time in the nursing program. Descriptive statistics of means and standard deviations (SD) of the measured items are shown in Table 1. Distributions of the items are shown a large majority of the responses are four or five, making the distributions of all items left-skewed (Table 1\&2).

The regression analysis Table 2 showed that the model explained $81.6 \%$ of the variance in the students' total learning experience. The results showed that 6 out of 7 items had a statistically significant effect on the students' learning concepts (at $\alpha<.05$ ) and these statistically significant items were positively related. The first and most influential item was "Lab experiences clarify the lecture material" ( $\mathrm{B}=0.297, \mathrm{P}<0.001$ ). The positive parameter estimates of 0.297 mean that if this item was increased (improved) by one unit, then the effectiveness of lab experiences in assisting students in learning concepts (DV) will increase (improve) by 0.297 unit, and all other parameter estimates can be interpreted in the same way. The second influential item was "The lab in this course has adequate facilities" ( $\mathrm{B}=0.188, \mathrm{P}<0.001)$, followed by the third most influential item, "The lab is a worthwhile part of the didactic course" $(\mathrm{B}=0.162, \mathrm{P}<0.001)$. "The nursing simulation lab procedures are clearly explained" $(\mathrm{B}=0.158, \mathrm{p}=$ 0.001) ranked as the fourth most influential item and "Instructor can answer my questions about what I should be doing in the lab" ( $B=0.134, P=0.013$ ) is the fifth most influential. The sixth and least influential item was "I am able to complete the lab activities in the time allotted" ( $\mathrm{B}=0.112, \mathrm{P}=0.008)$. The seventh item, "The lab sessions were well organized" in the "Lab evaluation tool" showed a negative relationship but was not statistically significant $(B=-0.06$, $P=0.166)$, therefore, the organization of the lab sessions were not considered as an influential item from the students' perspectives.

\section{Discussion}

This study aimed to examine factors that influence "students' learning of concepts in the simulation lab." Most factors tested in the simulation lab evaluation tool were significantly related to the students' overall lab experience indicating that the simulation lab is a rich area for student learning. Based on the study results, students were mostly concerned about how the simulation lab will prepare them to better understand the associated didactic course material and concepts which was reflected in the "Lab experiences clarify the lecture material" and "The content of the lab is a worthwhile part of this course" items. The results also indicated that students' learning experiences were affected by the availability and quality of simulation equipment used in the simulation lab, as indicated by the second influential factor in this study "The lab in this course has adequate facilities".

The clarity in explaining the nursing procedures and skills as demonstrated by the nursing instructor was the most influential factor in affecting the students' lab experience. The value of the lab experience as an important part of the didactic course also reflects how the didactic course objectives were met during the simulation lab experience. In this study, the importance of the content demonstrated during the simulation lab was the third most influential factor. Barth [17] argued, the clarity of instruction provided in the students' lab was included as an item on the survey. Barth [17] found the clarity item on the evaluation tool did coincide with the quality of instruction. In other words, there was an association with the outcome variable "quality of instruction." 
The instructor's quality of instruction as an item relates to the preparation of the instructor and how well the concepts were demonstrated in the simulation session. Barth [16] found that students who noticed the preparation of the instructor reported having better overall learning experiences. Thus, higher quality instruction will lead to higher student satisfaction in their learning experience. Schumacher [18] student's Health Education Systems, Inc. (HESI) examination (i.e.: a predictor exam for NCLEX-RN examination) was higher among students who had a combination of classroom and simulation teaching. This exam detected higher critical thinking abilities, increased students' self-confidence, and increased knowledge acquisition among students who had a simulation lab during their learning experience [4]. The lab experience allows instructors to link the objectives of the didactic courses to the simulated real-life scenarios.

Students rated the contribution of simulation lab in explaining the didactic course material (lab experiences clarify the lecture material) higher than the quality and availability of simulation equipment (the lab in this course has adequate facilities). This result agrees with Casida and Shpakoff [11] study in which students rated simulation labs as an effective learning experience for learning critical care nursing skills. This highlights the importance of simulation labs in evaluating the students' understanding of the concepts learned in the didactic courses and their application of the knowledge learned in making clinical judgments in a simulation environment that mimics the actual clinical settings [11].

This study supports the current trends of the importance of simulation as an effective approach for advancing nursing students clinical skills in a non-threatening learning environment. In this study, students rated "lab procedures are clearly explained to me" item as the fourth influential factor in the overall students' learning experience, indicating incredible importance of clear and concise skills demonstrations by instructors to optimize simulation effectiveness. Instructors play a major role in using students' lab time effectively to allow students to have hands-on experience to practice essential skills.

Simulation labs run by instructors had a preference over simulation labs run by teaching assistants in the nursing field [17]. In this study, students rated the guidance received from their instructor as the fifth influential factor which was reflected by the item "My instructor can answer my questions about what I should be doing in the lab". Luctkar-Flude et al. [17] compared students' preference on having an instructor to lead simulation labs versus teaching assistant (i.e. graduate students) and found students preferred simulations led by instructors as it provided them with the guidance needed for demonstrating nursing skills, clarifications of emerging questions, reinforcement of avoiding errors in the practical demonstration of the skills, realism, and problem-solving strategies in a collaborative way. This highlights the importance of emphasizing running the simulation labs by instructors [17]. Simulations led by instructors allow students to receive cues, ongoing feedback, and debriefing at the end of simulation which cultivates critical thinking. This supports the building of a supportive learning environment, helps reduce the students' anxiety level, and boosts the students' confidence level with their psychomotor skills and knowledge.

The effective use of students' learning time expended in the labs was ranked as the sixth most influential factor as indicated by "I am able to complete the lab activities in the time allotted" item. This indicates students are concerned about how their time is being managed by the instructor for a higher quality of learning outcomes and not that the instructor is wasting the student's time. In this study, the effective use of lab time in which it allows a time slot for students to practice their learned procedure had the lowest weight in affecting the students learning experience. However, it was a statistically significant factor and counted toward the total explained variance. This result shows that students are concerned about what concepts or skills they learn during each period in the simulation lab more than when it was allocated [4].

The organization of the clinical lab day provides the instructor and students with the best time management to achieve the objectives of each clinical day. The students' responses to the seventh influential factor, "The lab sessions are well organized", surprisingly indicated that the order and way in which the lab sessions were organized was of no interest to the students. This result was unexpected based on its level of importance for the nursing students. In this study, nursing students tend to value achieving the course objectives during the simulation time more than the order of activities scheduled for their simulation day. This result contradicts the literature results possibly because of the nature of clinical skills the students learn that differs in the level of importance to the students from didactic classes. However, this factor was not statistically significant in influencing the students learning experience. The importance of organizing course material was reflected in Barth [17] which found that the organization of the course material was a significant factor related to the quality of instruction in the didactic classes.

Students' evaluation of the simulation of clinical courses would provide a great source of information for effective curriculum planning if used effectively. Thus, it necessitates the use of a comprehensive tool that measures the three different aspects of students' learning experience which includes the cognitive, affective, and psychomotor dimensions of learning. Accurate student evaluation scores can assist nursing educators to build simulation lab course syllabi successfully based on the students learning perspectives and enhance the students' learning experience [1]. Other factors that measure the multidimensional aspects of the students' learning experience (e.g., the psychomotor, cognitive, and affective domains) should be addressed in the student's course evaluation tool $[1,11]$. In nursing, addressing the external learning 
outcome measures such as NCLEX-RN and the competency rating of the employer are other important aspects of nursing learning outcomes that should be included in the student course evaluation tools. Students' input on these factors can assist nursing educators in making decisions for curriculum changes and improving choices of teaching strategies.

The effective use of time management of the simulation lab represented a higher influential level for students' learning experience than the actual organization of tasks presented for each simulation lab. Students appreciated the prioritization of the skills learned and enforcing their knowledge and skills rather than having an organized fixed plan for their class. This indicates that meeting some versus all of the learning objectives during the assigned simulation time can help students achieve their learning needs more than having a set of objectives for a specific planned simulation time. Students rated their ability to demonstrate their skills during the simulation time as the last influential item. This reflects that students care about achieving their learning needs rather than demonstrating a procedure or a skill. This result agrees with the Luctkar-Flude et al. [12] study in which students preferred to have an instructor-led simulation where they can ask questions, receive cues from the instructor, and receive ongoing feedback and have a planned debriefing at the end of simulation sessions rather than having a step-organized simulation lab that is led by students in which the student-instructor will observe what the students are doing based on step-order during the lab and offer a debriefing at the end of the simulation session.

This study indicates simulation lab experience is important in enriching and explaining the concepts learned in nursing didactic courses and preparing students for providing effective care for patients at the clinical sites. Students' evaluation of simulation provided input about factors that can affect how and what students are learning during simulation time. Using students' input in the evaluation of the simulation lab will help nurse educators strengthen the nursing curriculum and make necessary changes to meet the course objectives, students' needs, and external learning outcomes.

\section{Study Limitations}

The tool used for collecting students' evaluation of simulation labs was short. Due to the retrospective nature of the data collection method of simulation course evaluation, the qualitative information on students' perspectives was not available for analysis. Thus, the students' interpretation of the items listed and their description of the simulation experience was not addressed. The demographics and the background of the simulation facilitator were not available to compare between instructor-led and student-led simulations. Besides, the course information such as the name of the course and the level of students enrolled in it was not available to compare the effectiveness of simulations in different nursing courses. Due to the non-random nature of our sample, the study results cannot be generalized.

\section{Conclusion and Recommendation}

Simulation provides students with a non-threatening learning environment that can boost students' confidence and clinical judgment. This study revealed multiple factors can affect the students learning experience. Addressing these factors based on the students' perspectives can help nursing educators plan appropriate curriculum changes to match the students' and nursing program's learning outcomes. In this study, the most influential factors in students' evaluation of their learning experiences were the congruency between didactic courses and simulations, the availability of quality supplies, and the clarity of simulation and instructors preparedness. Nursing faculty need to continue improving the simulation lab experience by addressing the students' feedback at the end of semester evaluation of simulation course. Instructors also need to provide continuous monitoring for the effectiveness of their teaching practices by using student evaluation (SET) of simulation results in planning their future simulation classes. Student evaluation of simulation is considered an important and efficient way of learning about students' perspectives toward lab activities and enhancing the quality of nursing student learning experiences. Building improvement plans based on the students' perspective in students' evaluations will enable instructors to teach more efficiently. Future studies need to look at the narrative students' evaluation of simulation experience and analyze it based on categories that relate to the course outcomes. To use the student evaluation as a useful tool in teaching, instructors need to reflect on their belief of the course outcomes and make changes to allow students to build their conceptual understanding of the material.

\section{Source of Funding}

The authors have not received any form of funding to prepare this manuscript.

\section{Ethical Clearance}

All procedures followed were per the ethical standards of the responsible committee on human experimentation (institutional and national) and with the Helsinki Declaration of 1975, as revised in 2000 .

\section{Conflict of Interest}

The authors declare no conflict of interest.

\section{Acknowledgement}

None.

\section{References}

1. Jeffreys M, Massoni M, O Donnell M, Smodlaka I (1997) Student evaluation of courses: Determining the reliability and validity of three survey instruments. J Nurs Educ 36(8): 397-400.

2. American Association of Colleges of Nursing (AACN) Home.

3. Lapkin S, Levett Jones T, Bellchambers H, Fernandez R (2010) Effectiveness of Patient Simulation Manikins in Teaching Clinical Reasoning Skills to Undergraduate Nursing Students: A Systematic Review. JBI Libr Syst Rev 8(16): 661-694. 
4. Lasater K (2007) High fidelity simulation and the development of clinical judgment: Students' experiences. J Nurs Educ 46(6): 269-276.

5. Rauen C (2004) Simulations as a teaching strategy for nursing education and orientation in cardiac surgery. Crit Care Nurse 24(3): 46-51.

6. Thornton G, Mueller Hanson R (2004) Developing Organizational Simulations: A Guide for Practitioners and Students. Mahwah, NJ: Lawrence Erlbaum.

7. Swenty C, Eggleston B (2011) The Evaluation of Simulation in Baccalaureate Nursing Program. Clin Simul Nurs 7(5): e181-e187.

8. Portsman S, Schumaker L, Hamilton P (2009) Evaluating the impact of scenario-based high-fidelity patient simulation on academic metrics of student success. Nurs Educ Perspect 32(4): 259-265.

9. Frey M (2012) Clinical simulation labs in the real world. RT: The Journal for Respiratory Care Practitioners 25(11): 22-24.

10. Knudson L (2013) Integrating simulation into student learning experiences. AORN J 97(4): C5-C6.

11. Casida J, Shpakoff L (2012) Baccalaureate Student Perceptions of Integrating Simulation as a Teaching Strategy in an Acute and Critical Care Nursing Course. Clinical Simulation in Nursing 8(8): e347-e352.
12. Luctkar Flude M, Wilson Keates B, Tyerman J, Larocque M, Brown C (2017) Comparing Instructor-Led Versus Student-Led Simulation Facilitation Methods for Novice Nursing Students. Clin Simul Nurs 13(6): 264-269.

13. Kim M, Kim S (2017) Debriefing Practices in Simulation-Based Nursing Education in South Korea. Clinical Simulation in Nursing 13(5): 201-209.

14. Teixeira C, Pereira M, Kusumota L, Gaioso V, Mello C, et al. (2015) Evaluation of nursing students about learning with clinical simulation. Rev Bras Enferm 68(2): 284-291.

15. Lewis D, Ciak A (2011) The impact of a simulation lab experience for nursing students. Nurs Educ Perspect 32(4): 256-258.

16. Stanford University (1997) Using student evaluations to improve teaching. Stanford University Newsletter on Teaching: Speaking of Teaching.

17. Barth M (2010) Deciphering Student Evaluations of Teaching: A Factor Analysis Approach. Journal of Education for Business 84(1): 40-46.

18. Schumacher L (2004) The impact of using high-fidelity computer simulation on critical thinking abilities and learning outcomes in undergraduate nursing students. Dissertation Abstracts International 65(10b). 